\title{
Imprints of Nonextensivity in Multiparticle Production*
}

\author{
G.Wilk ${ }^{\dagger \dagger}$ and Z.Włodarczyk ${ }^{2} \ddagger$ \\ ${ }^{1}$ The Andrzej Soltan Institute for Nuclear Studies \\ Hoża 69; 00-689 Warsaw, Poland \\ ${ }^{2}$ Institute of Physics, Pedagogical University \\ Konopnickiej 15; 25-405 Kielce, Poland
}

October 22, 2018

\begin{abstract}
The statistical methods based on the classical Boltzmann-Gibbs (BG) approach are at heart of essentially all descriptions of multiparticle production processes. In many cases, however, one observes some deviations from the expected behaviour. It is also known that conditions necessary for the BG statistics to apply are usually satisfied only approximately. Two attitudes are possible in such situations: either to abandon statistical approach trying some other model or to generalise it to the so called nonextensive statistics (widely used in the similar circumstances in many other branches of physics). We shall provide here an overview of possible imprints of non-extensitivity existing both in high energy cosmic ray physics and in multiparticle production processes in hadronic collisions, in particular in heavy ion collisions.
\end{abstract}

PACS numbers: 05.40.Fb 24.60.-k 05.10.Gg

Keywords: High energy multiparticle production, Nonextensive statistics, Thermal models

\section{Introduction}

The high energy collisions are usually connected with production of large number of secondaries (mostly $\pi$ and $K$ mesons). The strong interactions involved here make their detail descriprion from first principles impossible and one is forced to turn to phenomenological models of various kinds. The statistical models were the first successful approaches to the multiparticle production processes since the beginning of the subject almost half century ago [1] and they remain still very much alive today, especially in all analysis of multiparticle data performed from the point of view of the possible formation of the new state of matter - the Quark Gluon Plasma (QGP) [2]. They

\footnotetext{
${ }^{*}$ Invited talk presented by G.Wilk at $6^{\text {th }}$ International Workshop on Relativistic Aspects of Nuclear Physics (RANP2000), Caraguatatuba, Tabatinga Beach, São Paulo, Brazil, October 17-20, 2000.

†e-mail: wilk@fuw.edu.pl

${ }^{\ddagger}$--mail: wlod@pu.kielce.pl
} 
are all based on the Boltzmann-Gibbs form of entropy, which is identical to the so called Shannon entropy used in the information theory approach.

We would like to stress at this point that information theory can be also applied to hadronic processes [3]. Its importance there is best visualized by the following example. Suppose that some experiment provides us with data on a limited set of $n$ observables: $R_{1}, R_{2}, \ldots, R_{n}$. This triggers the theory, which follows with a variety of models, usually completely different (if not contradictory) but each claiming good agreement with these data. The models provide therefore apparently different views of the same physical reality (data) leading to confusion on what is actually going on. It happens this way because experimental data contain only limited amount of information to which theoretical models add their own specific assumptions born not in data but in our minds. To quantify this situation one must find the way to answer (in a model independent way) the following question: given physical assumptions of a model (plus phase space and conservation laws), what are their most trivial consequences? This can be done only be by resorting to information theory which defines triviality as the lack of information (the less information the more trivial). The measure of information provided by Shannon entropy (or some other kind of entropy, in our talk this will be Tsallis entropy [4]) allows us then to quantify the whole problem.

Let us elaborate this point a bit further. Experiment gives us usually set of points to which one tries to fit some distribution function $\rho(x)$, which can be thought to be some probability distribution. In this case Shannon entropy corresponding to it is

$$
S=-\int \rho(x) \ln [\rho(x)] .
$$

We are looking for the most trivial $\rho(x)$, i.e., for one containing the least information, and it means for such, which informational entropy is maximal under given circumstances (constraints) W. What are those constraints? At first, being a probability distribution $\rho(x)$ must be non-negative and normalized, i.e.,

$$
\int \rho(x) d x=1
$$

Further, $\rho(x)$ has to reproduce the experimental results given in the form of expectation values $\left\langle R^{(k)(x)}\right\rangle=R_{k}$ of measured set of observables $R^{(k)}$, i.e.,

$$
\int d x R^{(k)}(x) \rho(x)=R_{k} ; k=1, \ldots, m .
$$

Using eqs.(2) and (3) as constraints one then maximalizes information entropy (11) and as results one gets the seek for distribution $\rho(x)$,

$$
\rho(x)=\frac{1}{Z} \cdot \exp \left[-\sum_{k=1}^{m} \beta_{k} R^{(k)}(x)\right],
$$

where $Z$ is calculated from the normalization condition. This distribution reproduces known information (i.e., it tells us the truth, the whole truth about experiment) and contains the least information (i.e., it tells us nothing but the truth). In this sense it is the unique, most plausible (least biased), model independent probability distribution describing the outcomes of our

\footnotetext{
${ }^{1}$ One should remember that formula (1) assumes that $\rho(x)$ is measured against some other distribution $\rho_{0}(x)$ corresponding to equal a priori probability being assigned to equal volumes of phase space. This is sometimes called

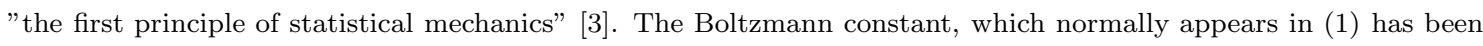
henceforth assumed unity, $k_{B}=1$.
} 
experiment. The Lagrange multipliers $\beta_{k}$ are given as solutions of the constraint equations and $R_{k}=\partial \ln Z\left(\beta_{1}, \ldots, \beta_{m}\right) / \partial \beta_{k}$.

Now we can understand why apparently completely disparate models can describe sucessfully the same experiment. It is because they share (usually in an implicite than explicite way) the same set of basic common assumptions (i.e., constraints $R_{k}$ ) and, at the same time, they differ completely in the rest of them, i.e., in those $R_{k}$, which contain assumptions particular for a given model (and which are completely not relevant to its agreement with experiment)].

This example tells us that statistical approach is not necessarily connected with any thermodynamical model but has, in fact, much broader range of applications. In our example above the formula (4) is identical with formulas used in thermal models [2] but parameters $\beta_{k}$ can take any values depending on the energy and multiplicity of the event under considerations [3]. It is worth to remind at this point the very old observation [6] that exponential form (4) of so many distributions observed experimentally can have its origin in the fact that the actual measurements extend only to subsystems of a total system. The summation over the unmeasured (i.e., essentially overaged over) degrees of freedom introduces the randomnization, which can be most economically described as a kind of thermal bath leading to thermal-like exponential distributions with some effective "temperature " $T$.

In this approach dynamics lies in our ability to reproduce the known information (i.e., is hidden in $\beta_{k}$ ). However, the big advantage of this method is that it prevents us from smuggling in anything additional, not being present in data. The outcome depends on the funtional form of the entropy. The Shannon form (1) is the most popular one because it coincides in thermodynamical limit with the Boltzmann-Gibbs (BG) form allowing for contact with standard thermodynamics. However, as newtonian mechanics is not eternal and universal (in the sense that in the limit of large velocities it must be replaced by special relativity, in the limit of vanishingly small masses quantum mechanical effects must be accounted for and in the limit of extremaly large masses (stars, galaxies,...) it must be replaced by general relativity) the same can be said about the classical BG statistical mechanics. It is fully satisfactory only if

- effective microscopic interactions are short-ranged (i.e., we have close spacial connections);

- effective microscopic memory is short-ranged (i.e, we have close time connections);

- boundary conditions are non (multi)fractal (i.e., the relevant space-time and/or phase space is non (multi)fractal).

As a matter of fact despite the popularity of BG it seems that in majority of situations we are dealing with, at least one of these conditions is not satisfied. Therefore it is justified to look for the possible generalization of $\mathrm{BG}$ which would provide a compactifications of all possible deviations from the ideal BG statistics into a minimal - possible only one - additional parameters. In this

\footnotetext{
${ }^{2}$ A completely forgotten example of such situation is discovery that to explain all experimental data on the multiparticle reactions of that time it is only necessary to assume that the phase space is effectively one-dimensional (i.e., the $p_{T}$ cut-off) and that not the whole initial energy $\sqrt{s}$ of reaction is used for the particle production (i.e., the existence of the inelasticity $K$ or of the leading particle effect).
} 
way we arrive at the notion of Tsallis statistics based on Tsallis entropy 佨:

$$
S_{q}=-\frac{1-\int d x \rho(x)^{q}}{1-q} .
$$

We shall not dwell too much on it here as this is the subject of separate lecture by Prof. Tsallis himself [7]. The only points we would like to stress here for our purposes are:

- for $q \rightarrow 1$ one recovers the usual Shannon entropy form of (11);

- with the normalization condition imposed on $\rho(x)$ as before and with the new definition of the mean values, $\langle R\rangle_{q}=\int d x R(x) \rho(x)^{q}$, one replaces formula (4) with

$$
\rho(x)=\frac{1}{Z} \cdot\left[1-(1-q) \sum_{k=1}^{m} \beta_{k} R^{(k)}(x)\right]^{\frac{1}{1-q}} ;
$$

(notice that, again, for $q \rightarrow 1$ (6) goes to (何). As before, this distribution reproduces known information and conveys least information but this time information is measured in a different way, which is paramatrized by the parameter $q)$;

- the resultant staistics is non-extensive in the sense that for two subsets $A$ and $B$ such that probabilities $p_{i j}(A+B)=p_{i}(A) p_{k}(B)$ the entropy is not additive but instead

$$
S_{q}(A+B)=S_{q}(A)+S_{q}(B)+(1-q) S_{q}(A) S_{q}(B)
$$

and additivity is recovered only in the limit $q \rightarrow 1$;

- the whole Legendre structure of thermodynamics is preserved for $q \neq 1$ [四, 7].

Although the nonextensive statistics were used for a long time in many branches of physics 《], their possible imprints in multiparticle production are very fresh. In this talk we shall concentrate on some cosmic ray experiments and accelerator experiments, which provided us with examples of a nonextensive behaviour. Both will demonstrate traces of nonextensivity seen as nonexponentiality of some distributions, i.e., where $\exp (x) \Rightarrow \exp _{q}(q)=[1-(1-q) x]^{1 /(1-q)}$. At the end we shall, however, list all other works in which one can find similar conclusions [9.

\section{Cosmic Ray Example}

Part of the cosmic ray detector system consists of extrathick lead emulsion chambers. Such chamber is build with a number of sandwiches of X-ray sensitive film, plate of lead and layer of emulsion (first allows to localize the point at which external particle of cosmic rays enters, second forces it to interact and to start the electromagnetic cascade process, and third allows to calculate deposited energy). In some experiments one observes that distribution of the points of first interaction (cascade starting points) does not follow the expected simple exponential rule [10]:

$$
\frac{d N}{d T}=\text { const } \cdot \exp \left(-\frac{T}{\lambda}\right)
$$

\footnotetext{
${ }^{3}$ There is a formalism, which expresses both the Tsallis entropy and the expectation value using the escort probability distribution, $P_{i}=p_{i}^{q} / \sum p_{i}^{q}$. 4 . However, it is known [8] that such an approach is different from the present nonextensive formalism since the Tsallis entropy expressed in terms of the escort probability distribution has a difficulty with the property of concavity.

${ }^{4}$ Here $N$ is the number of cascades originating at depth $T$ expressed in the so called cascade units where 1 c.u. $=6.4 \mathrm{~g} / \mathrm{cm}^{2}=0.56 \mathrm{~cm}$ of $\mathrm{Pb}$ and $\lambda$ is the mean free path for the $\mathrm{p}-\mathrm{Pb}$ interacions.
} 
but deviates from it noticeably for larger $T$. This phenomenon acquired even its own name: long flying component (because original hadrons tend to fly longer without interaction than naively expected).

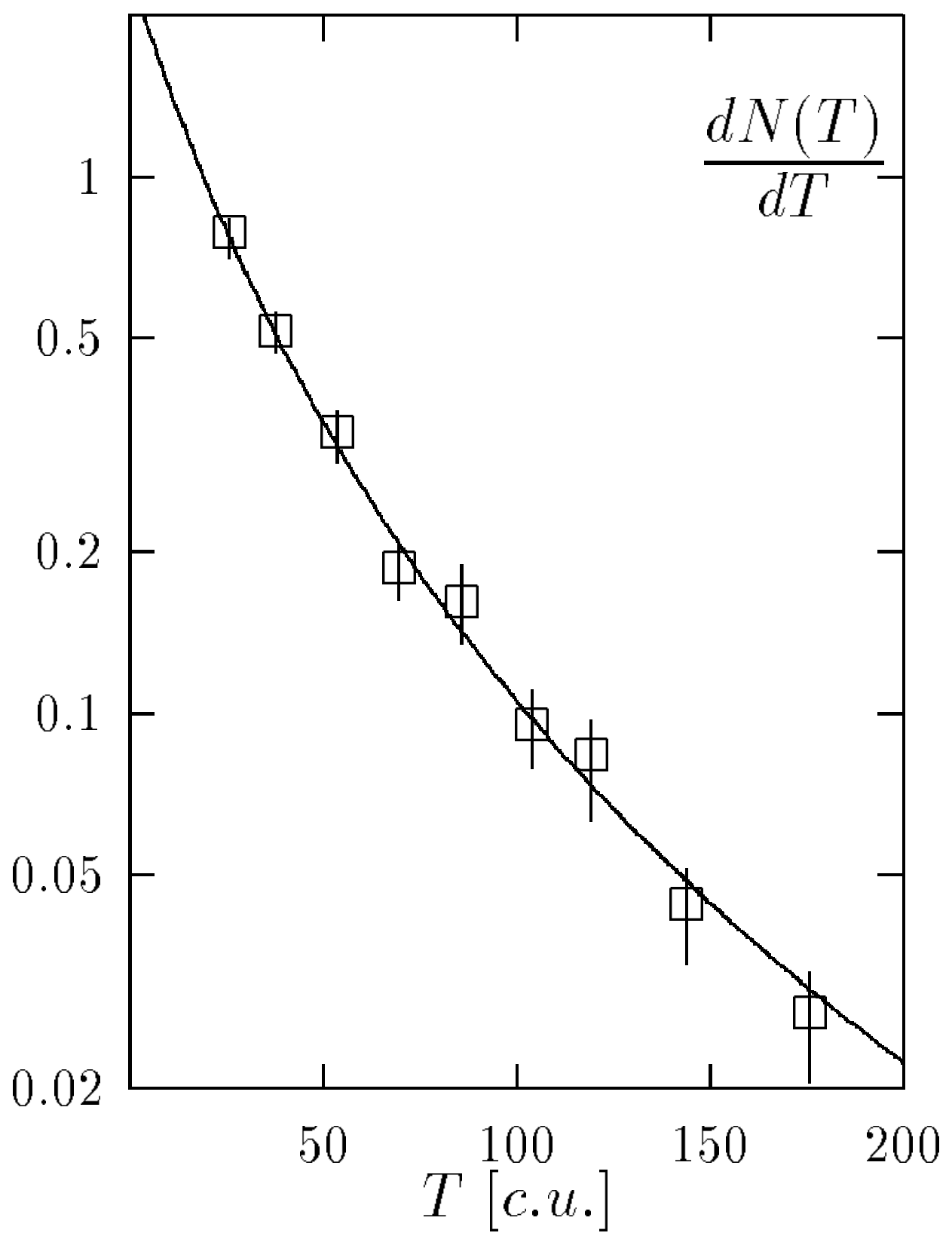

Fig. 1 Depth distribution of the starting points, $d N(T) / d T$, of cascades in Pamir lead chamber. Notice the non-exponential behaviour of data points (for their origin cf. [10]) which can be fitted by Tsallis distribution (10) with $q=1.3$. (This figure is reproduced from Fig. 1 of [11]).

In [10] we have argued that the observed effect can be just another manifestation of the fluctuation of the corresponding hadronic cross section $\sigma=A m_{N} \frac{1}{\lambda}$ (where $A$ denotes the mass number 
of the target and $m_{N}$ is the mass of the nucleon, such a possibility is widely discussed in the literature and observed in diffraction dissociation experiments on accelerators, cf. [10] for details and references). As was shown in [10] such fluctuations of cross section (i.e., in effect $1 / \lambda$ ) with relative variance

$$
\omega=\frac{\left\langle\sigma^{2}\right\rangle-\langle\sigma\rangle^{2}}{\langle\sigma\rangle^{2}} \geq 0.2
$$

allow to describe the observed effect. However, it turns out [11] that the same data can be fitted by the nonextensive formula

$$
\frac{d N}{d T}=\text { const } \cdot\left[1-(1-q) \frac{T}{\lambda}\right]^{\frac{1}{1-q}}
$$

with parameter $q=1.3$ (in both cases $\lambda=18.85 \pm 0.66$ in c.u. defined above), cf. Fig. 1 .

This example provides us hint on the possible physical meaning of the nonextensvity parameter $q$. Comparing both explanations one can suspect that perhaps, at least in this case, it has something to do with the fluctuations of the seemingly constant parameter $1 / \lambda$ in the exponential formula (8). We shall argue later that this is indeed the case but before let us demonstrate another example of this kind, now from the high energy multiparticle production processes domain.

\section{Heavy ion collision example}

Heavy ion collisions are of particular interest at present because they are the only place were one expects the possible formation of the QGP - a new state of matter [2]. The termal and/or all kind of statistical models are playing very important role here because one of the crucial parameter is the temperature $T$ of reaction deduced usualy from the transverse momentum distributions, $d N / d p_{T}$. This is, however, valid procedure only when $d N / d p_{T}$ is of exponential form. Therefore any deviation from such behaviour are always under detailed scrutiny in which one is searching for the possible causes. In [12] it was suggested that the extreme conditions of high density and temperature occuring in ultrarelativistic heavy ion collisions can lead to memory effects and longrange colour interactions and to the presence of non-Markovian processes in the corresponding kinetic equations 13]. Actually, as has been shown in [12, 14], $d N\left(p_{T}\right) / d p_{T}$ are best described, cf., Fig. 2, by a slightly nonexponential function of the type

$$
\frac{d N\left(p_{T}\right)}{d p_{T}}=\text { const } \cdot\left[1-(1-q) \frac{\sqrt{m^{2}+p_{T}^{2}}}{k T}\right]^{\frac{1}{1-q}} \quad \stackrel{q \rightarrow 1}{\Longrightarrow} \quad \text { const } \cdot \exp \left(-\frac{\sqrt{m^{2}+p_{T}^{2}}}{k T}\right) .
$$

Here $m$ is the mass of produced particle, $k$ is the Boltzmann constant (which we shall, in what follows, put equal unity) and $T$ is, for the $q=1$ case, the temperature of the reaction considered (or, rather, the temperature of the hadronic system produced). The deviation from the exponential form one finds is very small, on the level $q=1.015$. However, as we shall demonstrate later it can lead to a quite dramatic effects. It was also shown in [12] that to first order in $|q-1|$ the generalized slope becomes the quantity

$$
T_{q}=T+(q-1) m_{T} .
$$

with $T$ being temperature of a purely thermal source. This should be contrasted with the empirical relation for the slope parameter $T$, from which the freeze-out temperature (at which hadrons are 
created from the QGP) $T_{f}$ is then deduced,

$$
T=T_{f}+m\left\langle v_{\perp}\right\rangle^{2} .
$$

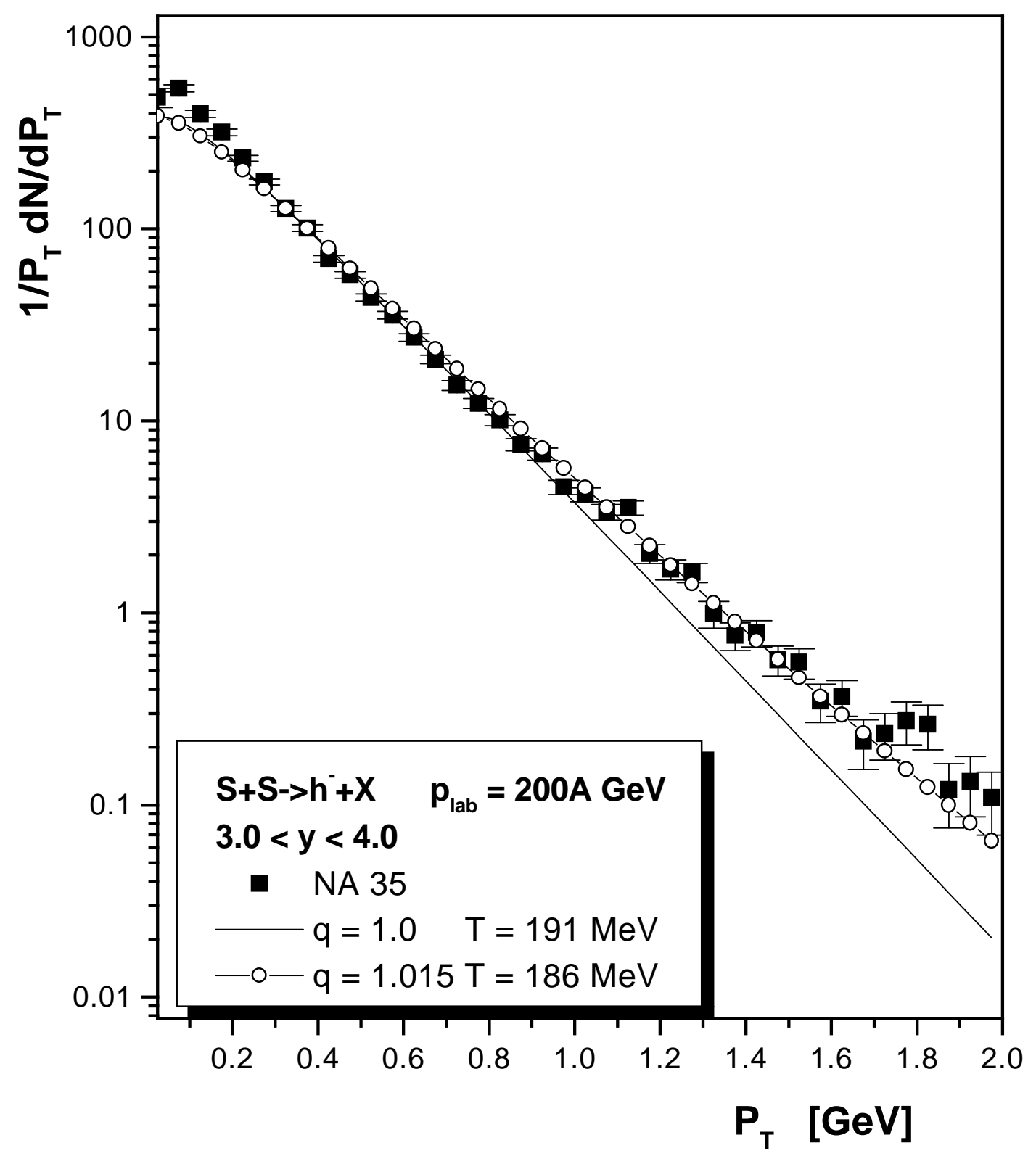

Fig. 2 The results for $p_{T}$ distribution $d N\left(p_{T}\right) / d p_{T}$ : notice that $q=1.015$ results describes also the tail of distribution not fitted by the conventional exponent (i.e., $q=1$ ). This figure is reproduced from Fig. 3 of [14].

The $\left\langle v_{\perp}\right\rangle$ is a fit parameter usually identified with the average collective (transverse) flow velocity of the hadrons being produced. In (12) one has, instead, a purely thermal source experiencing a kind of blue shift at high $m_{T}$ (actually increasing with $m_{T}$ ). The nonextensivity parameter $q$ 
accounts here for all possibilities one can find in [13] and could therefore be regarded as a new way of presenting experimental results with $q \neq 1$, signaling that there is something going on in the collision that prevents it from being exactly thermal-like in the ordinary sense mentioned above.

To the same cathegory belongs also analysis of the equilibrium distribution of heavy quarks in Fokker-Planck dynamics expected in heavy ion collisions [15. Not going into details in this case, we shall only mention that it was found that thermalization of charmed quarks in a QGP surroundings proceeding via collisions with light quarks and gluons results in a spectral shape, which can be described only in $q$ statistics with $\mathrm{q}=1.114$ (for $m_{C}=1.5 \mathrm{GeV}$ and temperature of thermal gluons $T_{g}=500 \mathrm{GeV}$ ).

\section{$4 \quad$ Fluctuations as a cause of nonextensivity?}

Guided by the cosmic ray example we would like to discuss now the hypothesis that the examples of nonextensivity in which exponential distribution becomes a power-like (Lévy type) distributions is caused by the fluctuations in the system which were not accounted for and which make $q \neq 1$ [16, 9]. In other words, $q$ summarizes the action of averaging over $1 / \lambda$ :

$$
\left\langle\exp \left[-\left(\frac{1}{\lambda}\right) \varepsilon\right]\right\rangle \Longrightarrow\left[1-(1-q) \frac{1}{\lambda_{0}} \varepsilon\right]^{\frac{1}{1-q}},
$$

where $1 / \lambda_{0}=\langle 1 / \lambda\rangle$. This connection, which is clear in the cosmic ray example, should also be true for the heavy ion collision. Actually, this later case is even more important and interesting because of the long and still vivid discussion on the possible dynamics of temperature fluctuations [17] and because of its connection with the problem of QGP production in heavy ion collisions [18].

Actually the conjecture (14) is known in the literature as Hilhorst integral formula [19] but was never used in the present context. It says that (for $q>1$ case, where $\varepsilon \in(0, \infty)$ )

$$
\left(1+\frac{\varepsilon}{\lambda_{0}} \frac{1}{\alpha}\right)^{-a}=\frac{1}{\Gamma(\alpha)} \int_{0}^{\infty} d \eta \eta^{\alpha-1} \exp \left[-\eta\left(1+\frac{\varepsilon}{\lambda_{0}} \frac{1}{\alpha}\right)\right] .
$$

where $\alpha=\frac{1}{q-1}$. Changing variables under the integral to $\eta=\alpha \frac{\lambda_{0}}{\lambda}$, one obtains

$$
L_{q>1}\left(\varepsilon ; \lambda_{0}\right)=C_{q}\left(1+\frac{\varepsilon}{\lambda_{0}} \frac{1}{\alpha}\right)^{-a}=C_{q} \int_{0}^{\infty} \exp \left(-\frac{\varepsilon}{\lambda}\right) f\left(\frac{1}{\lambda}\right) d\left(\frac{1}{\lambda}\right)
$$

where $f(1 / \lambda)$ is given by the following gamma distribution:

$$
f_{q>1}\left(\frac{1}{\lambda}\right)=f_{\alpha}\left(\frac{1}{\lambda}, \frac{1}{\lambda_{0}}\right)=\frac{\mu}{\Gamma(\alpha)}\left(\frac{\mu}{\lambda}\right)^{\alpha-1} \exp \left(-\frac{\mu}{\lambda}\right)
$$

with $\mu=\alpha \lambda_{0}$ and with mean value and variation in the form:

$$
\left\langle\frac{1}{\lambda}\right\rangle=\frac{1}{\lambda_{0}} \quad \text { and } \quad\left\langle\left(\frac{1}{\lambda}\right)^{2}\right\rangle-\left\langle\frac{1}{\lambda}\right\rangle^{2}=\frac{1}{\alpha \lambda_{0}^{2}}
$$

Notice that, with increasing $\alpha$ the variance (18) decreases and asymptotically (for $\alpha \rightarrow \infty$, i.e, for $q \rightarrow 1$ ) the gamma distribution (17) becomes a delta function, $f_{q>1}(1 / \lambda)=\delta\left(\lambda-\lambda_{0}\right)$. The relative variance for this distribution is given by

$$
\omega=\frac{\left\langle\left(\frac{1}{\lambda}\right)^{2}\right\rangle-\left\langle\frac{1}{\lambda}\right\rangle^{2}}{\left\langle\frac{1}{\lambda}\right\rangle^{2}}=\frac{1}{\alpha}=q-1 .
$$


For the $q<1$ case $\varepsilon$ is limited to $\varepsilon \in\left[0, \lambda_{0} /(1-q)\right]$. Proceeding in the same way as before, i.e., making use of the following representation of the Euler gamma function (where $\alpha^{\prime}=-\alpha=\frac{1}{1-q}$ )

$$
\left[1-\frac{\varepsilon}{\alpha^{\prime} \lambda_{0}}\right]^{\alpha^{\prime}}=\left(\frac{\alpha^{\prime} \lambda_{0}}{\alpha^{\prime} \lambda_{0}-\varepsilon}\right)^{-\alpha^{\prime}}=\frac{1}{\Gamma\left(\alpha^{\prime}\right)} \int_{0}^{\infty} d \eta \eta^{\alpha^{\prime}-1} \exp \left[-\eta\left(1+\frac{\varepsilon}{\alpha^{\prime} \lambda_{0}-\varepsilon}\right)\right]
$$

and changing variables under the integral to $\eta=\frac{\alpha^{\prime} \lambda_{0}-\varepsilon}{\lambda}$, we obtain $L_{q<1}\left(\varepsilon ; \lambda_{0}\right)$ in the form of eq. (16) but with $\alpha \rightarrow-\alpha^{\prime}$ and with the respective $f(1 / \lambda)=f_{q<1}(1 / \lambda)$ given now by the same gamma distribution as in (17) but this time with $\alpha \rightarrow \alpha^{\prime}$ and $\mu=\mu(\varepsilon)=\alpha^{\prime} \lambda_{0}-\varepsilon$. Contrary to the $q>1$ case, this time the fluctuations depend on the value of the variable in question, i.e., the mean value and variance are now both $\varepsilon$-dependent:

$$
\left\langle\frac{1}{\lambda}\right\rangle=\frac{1}{\lambda_{0}-\frac{\varepsilon}{\alpha^{\prime}}} \quad \text { and } \quad\left\langle\left(\frac{1}{\lambda}\right)^{2}\right\rangle-\left\langle\frac{1}{\lambda}\right\rangle^{2}=\frac{1}{\alpha^{\prime}} \cdot \frac{1}{\left(\lambda_{0}-\frac{\varepsilon}{\alpha^{\prime}}\right)^{2}}
$$

However, the relative variance

$$
\omega=\frac{\left\langle\left(\frac{1}{\lambda}\right)^{2}\right\rangle-\left\langle\frac{1}{\lambda}\right\rangle^{2}}{\left\langle\frac{1}{\lambda}\right\rangle^{2}}=\frac{1}{\alpha^{\prime}}=1-q
$$

remains $\varepsilon$-independent and depends only on the parameter $q$. As above the resulting gamma distribution becomes a delta function, $f_{q<1}(1 / \lambda)=\delta\left(\lambda-\lambda_{0}\right)$, for $\alpha^{\prime} \rightarrow \infty$, i.e., for $q \rightarrow 1$.

Summarizing: one can indeed say that the nonextensivity parameter $q$ in the $L_{q}(\varepsilon)$ distributions can be expressed by the relative variance $\omega$ of fluctuations of the parameter $1 / \lambda$ in the distribution $L_{q=1}(\varepsilon)$ :

$$
q=1 \pm \omega \quad \text { for } \quad q>1(+) \text { and } \quad q<1(-)
$$

Actually the above result (23) is derived for the particular (Gamma-like) shape of the fluctuation distribution function. How can it be realized? To answer this question let us notice [16, 9] that in the case when stochastic variable $\lambda$ is decribed by the usual Langevin equation

$$
\frac{d \lambda}{d t}+\left[\frac{1}{\tau}+\xi(t)\right] \lambda=\phi=\text { const }>0
$$

(with damping constant $\tau$ and source term $\phi_{q<1}=\frac{1}{\tau}\left(\lambda_{0}-\frac{\varepsilon}{\alpha^{\prime}}\right)$ or $\phi=\phi_{q>1}=\frac{\lambda_{0}}{\tau}$ ), then for the stochastic processes defined by the white gaussian noise form of $\xi(t)$ 局 one obtains the following Fokker-Plank equation [20] for the distribution function of the variable $\lambda$ :

$$
\frac{d f(\lambda)}{d t}=-\frac{\partial}{\partial \lambda} K_{1} f(\lambda)+\frac{1}{2} \frac{\partial^{2}}{\partial \lambda^{2}} K_{2} f(\lambda),
$$

with intensity coefficients $K_{1,2}$ defined by eq.(24) and equal to (cf., 21):

$$
K_{1}(\lambda)=\phi-\frac{\lambda}{\tau}+D \lambda \quad \text { and } \quad K_{2}(\lambda)=2 D \lambda^{2} .
$$

\footnotetext{
${ }^{5}$ It means that ensemble mean $\langle\xi(t)\rangle=0$ and correlator (for sufficiently fast changes) $\langle\xi(t) \xi(t+\Delta t)\rangle=$ $2 D \delta(\Delta t)$. Constants $\tau$ and $D$ define, respectively, the mean time for changes and their variance by means of the following conditions: $\langle\lambda(t)\rangle=\lambda_{0} \exp \left(-\frac{t}{\tau}\right)$ and $\left\langle\lambda^{2}(t=\infty)\right\rangle=\frac{1}{2} D \tau$. Thermodynamical equilibrium is assumed here (i.e., $t>>\tau$, in which case the influence of the initial condition $\lambda_{0}$ vanishes and the mean squared of $\lambda$ has value corresponding to the state of equilibrium).
} 
Its stationary solution has precisely the Gamma-like form we are looking for:

$$
f(\lambda)=\frac{c}{K_{2}(\lambda)} \exp \left[2 \int_{0}^{\lambda} d \lambda^{\prime} \frac{K_{1}\left(\lambda^{\prime}\right)}{K_{2}\left(\lambda^{\prime}\right)}\right]=\frac{1}{\Gamma(\alpha)} \mu\left(\frac{\mu}{\lambda}\right)^{\alpha-1} \exp \left(-\frac{\mu}{\lambda}\right),
$$

with the constant $c$ defined by the normalization condition, $\int_{0}^{\infty} d(1 / \lambda) f(1 / \lambda)=1$. It depends on two parameters:

$$
\mu(\varepsilon)=\frac{\phi_{q}(\varepsilon)}{D} \quad \text { and } \quad \alpha_{q}=\frac{1}{\tau D},
$$

with $\phi_{q}=\phi_{q>1, q<1}$ and $\alpha_{q}=\left(\alpha, \alpha^{\prime}\right)$ for, respectively, $q>1$ and $q<1$. Therefore eq. (23) with $\omega=\frac{1}{\tau D}$ is, indeed, a sound possibility with (in the case discussed above) parameter of nonextensivity $q$ given by the parameter $D$ and by the damping constant $\tau$ describing the white noise.

\section{$5 \quad$ Back to heavy ion collisions}

With the possibility of such interpretation of parameter $q$ in mind we can now came back to the example of heavy ion collisions and see what are its consequences in this case. It is interesting to notice that the relatively small departure of $q$ from unity, $q-1 \simeq 0.015$ 12, 14, if interpreted in terms of the previous section, indicates that rather large relative fluctuations of temperature, of the order of $\Delta T / T \simeq 0.12$, exist in nuclear collisions. It could mean therefore that we are dealing here with some fluctuations existing in small parts of the system in respect to the whole system (according to interpretation of [22]) rather than with fluctuations of the event-by-event type in which, for large multiplicity $N$, fluctuations $\Delta T / T=0.06 / \sqrt{N}$ should be negligibly small [18]. This controversy could be, in principle, settled by detailed analyses of the event-by-event type. Already at present energies and nuclear targets (and the more so at the new accelerators for heavy ions like RHIC at Brookhaven, now commisioned, and LHC at CERN scheduled to be operational in the year 2006) one should be able to check whether the power-like $p_{T}$ distribution $d N\left(p_{T}\right) / d p_{T}$ occurs already at every event or only after averaging over all events. In the former case we would have a clear signal of thermal fluctuations of the type mentioned above. In the latter case one would have for each event a fixed $T$ value which would fluctuate from one event to another (most probably because different initial conditions are encountered in a given event).

One point must be clarified, however. The above conjecture rests on the stochastic equation (24). Can one expect such equation to govern the $T$ fluctuations? To ansewer this question let us turn once more to the fluctuations of temperature [17, 18, 22 discussed before, i.e., to $\lambda=T$. Suppose that we have a thermodynamic system, in a small (mentally separated) part of which the temperature fluctuates with $\Delta T \sim T$. Let $\lambda(t)$ describe stochastic changes of the temperature in time. If the mean temperature of the system is $\langle T\rangle=T_{0}$ then, as result of fluctuations in some small selected region, the actual temperature equals $T^{\prime}=T_{0}-\tau \xi(t) T$. The inevitable exchange of heat between this selected region and the rest of the system leads to the equilibration of the temperature and this process is described by the following equation [23:

$$
\frac{\partial T}{\partial t}-\frac{1}{\tau}\left(T^{\prime}-T\right)+\Omega_{q}=0,
$$

which is, indeed, of the type of eq. (24) (here $\Omega_{q<1}=\frac{\varepsilon}{\tau \alpha^{\prime}}$ and $\Omega_{q>1}=0$ ). This proves the plausability of what was said above and makes the event-by-even measurements of such phenomenon 
very interesting one.

\section{Other imprints of nonextensivity}

The above discussed examples do not exhaust the list of the possible imprints of nonextensivity in multiparticle production know (or thought of) at present. As it is impossible to review all of them here, we shall therefore only mention them.

In [12, 14 the possible effects of nonextensivity on the mean occupation numbers $n_{q}$ and on the event-by-event fluctuation phenomena have been discussed. It turns out, for example, that some charateristics of fluctuations are extremaly sensitive to even small departures of $q$ from unity. Such departure can easily mimick the existing correlations or the influence of resonances.

In [24] an interesting attempt was presented to fit the energy spectra in both the longitudinal and transverse momenta of particles produced in the $e^{+} e^{-}$annihilation processes at high energies using $q$-statistical model. In this way one can have energy independent temperature $T$ and nonextensivity parameter $q$ rising quickly with energy from $q=1$ to $q=1.2$ and reflecting long range correlations in the phase space arising in the hadronization process in which quarks and gluons combine together forming hadrons (actually, this observation has general validity and applies to all production processes discussed here as well). In similar spirit is the work [25] which attemts to generalized the so called Hagedorn model of multiparticle production to $q$-statistics.

To the extend to which self-organized criticality (SOC) is connected with nonextensivity 何 one should also mention here a very innovative (from the point of view of high energy collision) application of the concept of SOC to such processes [26].

The other two examples do not refer to Tsallis thermostatistics directly, nevertheless they are connected to it. First is the attempt to study, by using the formalism of quantum groups, the Bose-Einstein correlations between identical particles observed in multiparticle reactions [27], second are works on intermittency and multiparticle distributions using the so called Lévy stable distributions [28]. They belong, in some sense, to the domain of nonextensivity because, as was shown in [29], there is close correspondence between the deformation parameter of quantum groups used in 27 and the nonextensivity parameter $q$ of Tsallis statistics and there is also connection between Tsallis statistics and Lévy stable distributions [28]. Some traces of the possible nonextensive evolution of cascade type hadronization processes were also searched for in [30]. The quantum group approach 27, 29 could probably be a useful tool when studying delicate problem of interplay between QGP and hadrons produced from it. It is plausible that description in terms of $q$-deformed bosons (or the use of some kind of interpolating statistics) would lead to more general results than the simple use of nonextensive mean occupation numbers $\langle n\rangle_{q}$ discussed above (for which the only known practical description is limited to small deviations from nonextensivity only).

One should mention also attempts to use Lévy-type distributions to fit the development of the cosmic ray cascades to learn of how many descendat they contain 31 or the explanation of the Feynman scaling violation observed in multiparticle distributions in terms of the $q$ parameter 32 . 


\section{Summary}

To summarize, evidence is growing in favour of the view that the standart statistical model can be enlarged towards the nonstandart statistics and that by including one new parameter $q$ it allows to repoduce much broader set of data than it was done so far. It was demonstrated that $q$ is probably connected with the intrinsic fluctuations existing in the system under consideration which were previously not considered at all [. Let us close with the remarks that, as was shown in [34], one can also try to use power-like distributions discussed here to the new formulation of the quantum field theory (for example, in terms of lorenzian rather than gaussian path integrals, accounting in this manner for some intrinsic long range correlations impossible to dispose of and extremaly difficult to account for by other methods).

Acknowledgements: One of us (G.W.) would like to thank the organizers of the RANP2000 for support making his participation in this workshop possible. Authors are grateful to Prof. S.Abe for his valuable comments.

\section{References}

[1] L.D. Landau and S.Z. Bilenkij, Nuovo Cim. Suppl. 3 (1956) 15 and Collected Papers of L.D. Landau, ed. D. Ter Haar, Pergamon Press (1965), p. 569. This model is still in use even today for quick and straightforward estimations of some quantities of interest, cf., M.Gaździcki, $Z$. Phys. C66 (1995) 659.

[2] Cf., for example, proceedings of Quark Matter'99, Nucl. Phys. A525 (1999) and references therein.

[3] G.Wilk and Z.Włodarczyk, Phys. Rev. D43 (1991) 794. The early references to all previous applications of this approach to multiparticle production can be found here as well.

[4] C.Tsallis, J. Stat. Phys. 52 (1988) 479; cf. also C.Tsallis, cond-mat/0010150 (to appear in Chaos, Solitons and Fractals (2001). For updated bibliography on this subject see http://tsallis.cat.cbpf.br/biblio.htm. Its recent summary is provided in the special issue of Braz. J. Phys. 29 (No 1) (1999) (available also at http://sbf.if.usp.br/WWW_pages/Journals/BJP/Vol29/Num1/index.htm).

[5] Y.-A. Chao, Nucl. Phys. B40 (1972) 475.

[6] E.T. Jaynes, Phys. Rev. 106 (1957) 620; 108 (1957) 171; L.Van Hove, Z. Phys. C21 (1985) 93; C27 (1985) 135.

[7] Cf. lecture by C.Tsallis, this volume.

\footnotetext{
${ }^{6}$ Actually it is likely that the importance of fluctuations is more general than their applications to $q$-statistics only. A good example is work 33 showing how fluctuations of the vacuum can change the theoretically expected gaussian spectra in string models into the observed exponential one with a kind of effective "temperature".
} 
[8] S.Abe, Phys. Lett. A275 (2000) 250.

[9] Cf., for example, G.Wilk and Z.Włodarczyk, The imprints of nonextensive statistical mechanics in high energy collisions; hep-ph/0004250; to be published in Chaos, Solitons and Fractals (2001).

[10] G.Wilk and Z.Włodarczyk, Phys. Rev. D50 (1994) 2318.

[11] G.Wilk and Z.Włodarczyk, Nucl. Phys. B (Proc. Suppl.) A75 (1999) 191.

[12] W.M.Alberico, A.Lavagno and P.Quarati, Eur. Phys. J. C12 (2000) 499.

[13] S.Gavin, Nucl. Phys. B351 (1991) 561; J.Rau, Phys. Rev. D50 (1994) 6911; S.Schmidt et al., Phys. Rev. D59 (1999) 094005; H.Heiselberg and X.-N.Wang, Phys. Rev. D53 (1996) 1892; V.K.Magas et al., Phys. Lett. B459 (1999) 33; Cs.Anderlik et al., Phys. Rev. C59 (1999) 388, 3309; F.Grassi et al., Phys. Rev. C62 (2000) 044904; J.Hormuzdiar et al., Particle Multiplicities and Thermalization in High Energy Collisions, arXiv:nucl-th/0001044.

[14] O.V.Utyuzh, G.Wilk and Z.Włodarczyk, J. Phys. G26 (2000) L39.

[15] D.B.Walton and J.Rafelski, Phys. Rev. Lett. 84 (2000) 31.

[16] G.Wilk and Z.Włodarczyk, Phys. Rev. Lett. 84 (2000) 2770.

[17] T.C.P.Chui, D.R.Swanson, M.J.Adriaans, J.A.Nissen and J.A.Lipa, Phys. Rev. Lett. 69 (1992) 3005; C.Kittel, Physics Today 5 (1988) 93; B.B.Mandelbrot, Physics Today 1 (1989) 71; H.B.Prosper, Am. J. Phys. 61 (1993) 54; G.D.J.Phillires, Am. J. Phys. 52 (1984) 629.

[18] L.Stodolsky, Phys. Rev. Lett. 75 (1995) 1044; E.V.Shuryak, Phys. Lett. B423 (1998) 9; S.Mrówczyński, Phys. Lett. B430 (1998) 9.

[19] C.f., for example, S.Curilef, Z.Phys. B100 (1996) 433 and references therein.

[20] N.G. van Kampen, Stochastic Processes in Physics and Chemistry, Elsevier Science Pub. B.V., North-Holland, Amsterdam 1987 (Chapter VIII).

[21] C.A.Ahmatov, Y.E.Diakov and A.Tchirkin, Introduction to Statistical Radiophysics and Optics, Nauka, Moscow, 1981 (in Russian).

[22] L.D.Landau, I.M.Lifschitz, Course of Theoretical Physics: Statistical Physics, Pergmon Press, New York 1958.

[23] L.D.Landau and I.M.Lifschitz, Course of Theoretical Physics: Hydrodynamics, Pergamon Press, New York 1958 or Course of Theoretical Physics: Mechanics of Continuos Media, Pergamon Press, Oxford 1981.

[24] I.Bediaga, E.M.F.Curado and J.M.de Miranda, Physica A286 (2000) 156.

[25] C.Beck, Physica A286 (2000) 164.

[26] Meng Ta-chung, R.Rittel and Z.Yang, Phys. Rev. Lett. 82 (1999) 2044 and C.Boros et al., Phys. Rev. D61 (2000) 094010 (cf. also: hep-ph/0009034).

[27] D.V.Anchishkin, A.M.Gavrilik and N.Z.Iogorov, Eur. J. Phys. A7 (2000) 229 and Mod. Phys. Lett. 15 (2000) 1637. 
[28] Cf., for example, R.Peschanski, Nucl. Phys. B253 (1991) 225 or S.Hegyi, Phys. Lett. B387 (1996) 642 (and references therein).

[29] C.Tsallis, Phys. Lett. A195 (1994) 329. See also A.Lavagno and P.Narayana Swamy, Mod. Phys. Lett. 13 (1999) 961 and Phys. Rev. E61 (2000) 1218.

[30] O.V.Utyuzh, G.Wilk and Z.Włodarczyk, Czech J. Phys. 50/S2 (2000) 132.

[31] M.Rybczyński, Z.Włodarczyk and G.Wilk, Self-organized criticality in atmospheric cascades, presented at the XI ISVHECRI, Unicamp-Campinas-Brazil, July 17-21, 2000; hepph/0005216; to be published in Nucl. Phys. B (Proc. Suppl.).

[32] F.S.Navarra, O.V.Utyuzh, G.Wilk and Z.Włodarczyk, Violation of the Feynman scaling law as a manifestation of nonextensivity, presented at Chacaltaya Meeting on Cosmic Ray Physics, July 23-27, 2000, La Paz, Bolivia, to be published in Nuovo Cim. C; hep-ph/0009165.

[33] A.Białas, Phys. Lett. B466 (1999) 301.

[34] R.A.Treuman, Phys. Rev. E57 (1998) 5150; Europh. Lett. 48 (1999) 8 and Phys. Scripta 59 (1999) 19, 204. See also R.S.Mendes in review volume quoted in 凷, p. 66.

\section{Discussion}

Question by K.Werner: Concerning your example $d N / d p_{T}$ : this seems to be quite under control,large $p_{T}$ is perturbative QCD (power-like), small $p_{t}$ is soft, so you have a superposition of these two processes. There seems to be no need of these additional fluctuations.

Answer: Your proposition is just one of many around us. You are right that adding two known (in their domain of applicability) mechanisms will also fit data. But what we are saying here is that, perhaps, there is mechanism which would show itself in the intermediate region and which can be described by the nonextensive parameter $q$. And it can be experimentally checked in eventby-event type of analysis, as was presented above. This would answer the question is there a need for something or not. Besides, it should be stressed that using only one new parameter $q$ one can fit quite large (in [24] the whole) region of $p_{T}$.

Question by F.Grassi: Did you try to fit $p_{T}$ distributions for other type of particles than charged? Answer: No, at least not yet.

Question by T.Kodama: If $q$ represents the measure of fluctuations of ensemble, then it would also reflect in multiplicity distributions. However, value of $q$ is different for the $p_{T}$ distribution and multiplicity distribution. How do you interpret this?

Answer: It is difficult for me to comment because so far I have not seen such analysis of the multiplicity data. But if things are really as you say then, assuming that everything was done correctly, I would argue that multiplicity is global characteristic whereas $p_{T}$ is a local one. Therefore they are sensitive to different aspects of the underlying dynamics and resulting parameters $q$ could $a$ priori be different. One can also say that for $p_{T}$ distribution the situation is more clear as we are simple replacing here $\exp (\ldots)$ by $\exp _{q}(\ldots)$. Multiplicity distributions depend on $q$ in indirect way only. 